\title{
Masters of metabolism: Matthias Tschöp and Richard DiMarchi
}

n June 26, 2011, in San Diego, the Merrifield Award of the American Peptide Society was given to Richard DiMarchi of Indiana University. A day later, again in San Diego, the Outstanding Scientific Achievement Award of the American Diabetes Association was given to Matthias Tschöp of the University of Cincinnati. Those attending both lectures might have witnessed many similarities; the Tschöp and DiMarchi laboratories had been working as a single integrated unit across two universities for the last seven years, designing and optimizing new therapeutics for the treatment of diabetes and obesity (Figure 1).

JCI: How did the two of you start collaborating?

Tschöp: We met at the Eli Lilly Research Laboratories, where Richard led drug discovery as a group vice president, while I was a postdoctoral fellow. Years later, when I had moved back to the US from Germany and Richard had left Lilly to be a chair of chemistry at Indiana University, we reconnected - our labs suddenly were just a twohour drive apart.

JCI: What avenue of research do you think has the greatest potential to improve the outlook for the obesity epidemic?

Tschöp: It seems increasingly clear that more than one neuroendocrine signal may have to be modulated at the same time in order to achieve beneficial metabolic effects with curative potential. Richard and I have been working on a series of approaches in which we are combining two or three gastrointestinal hormones into a single molecule.
JCI: So are you both optimistic that we'll find a drug-based cure for metabolic syndrome?

DiMarchi: I am a perpetual optimist, supported by three decades of personal experiences. This kind of work requires steady progress made through contributions from many laboratories establishing a foundation for the discovery of transformative medicines. We believe that our work is contributing to the identification of a novel formula that might define a successful prescription for treatment.

Tschöp: More skeptical colleagues frequently point out to me that evolutionary pressures drove the development of redundant systems to efficiently ingest and store calories. My answer is always that reproduction is a pretty important requirement for the survival of the species too, but endocrinologists figured out how to interrupt it by tricking the brain into believing that there was already an ongoing pregnancy. We need to figure out how to trick the brain into believing that the stomach has been bypassed without actually cutting patients open.

JCI: Your work at Lilly and now at Indiana University is largely based on macromolecules and, in particular, peptides - in spite of the limitation that peptide-based drugs almost always require injection. What are the advantages?

DiMarchi: In many ways the peptides and proteins that have emerged as drugs are nature's medicines. They have a high speci-

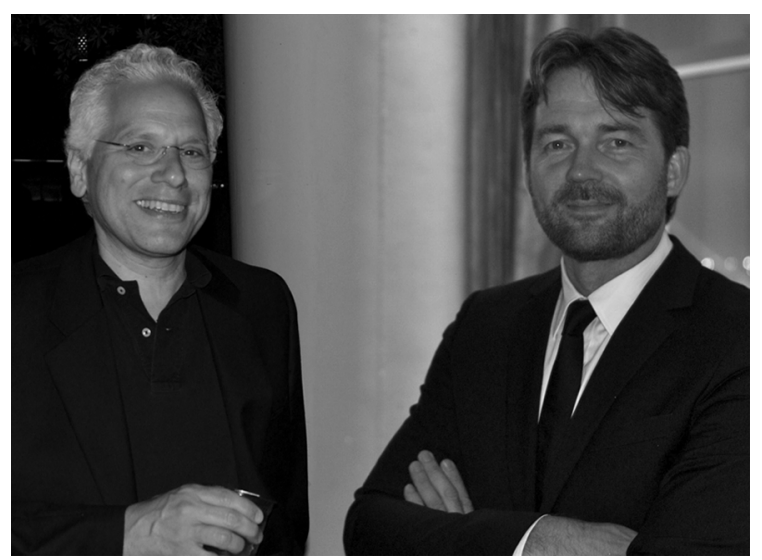

Figure 1

Richard DiMarchi (left) and Matthias Tschöp.

ficity of action with minimal off-target toxicity and natural routes of metabolic clearance. While injection is a limitation, the huge benefit is performance. However, it is important not to frame the question of drug discovery as an either/or option of conventional small molecules versus macromolecules. The combination of the two can deliver unprecedented efficacy with fewer adverse effects.

JCI: Given that you moved from pharma to academe, you're perhaps well positioned to answer the question of whether scientists will be successful in drug discovery outside of the pharmaceutical industry.

DiMarchi: Large organizations may be required for cost-efficient drug development and production and marketing of drugs. However, it is individual scientists who deliver breakthrough discoveries. A laboratory composed of broadly trained chemists and pharmacologists can make a huge difference, if properly funded and appropriately nurtured. I am quite certain that Bruce Merrifield, the father of solidphase chemical synthesis, could not have invented the concept if employed in a less supportive environment than an academic center like The Rockefeller University.

JCI: You're moving back to Germany this year. Will that be a challenge for your collaboration?

Tschöp: My wife and I are both from Munich, so when the Munich Hemholtz Centre together with the Technical University and the Ludwig Maximilians University Munich invited us to help build a new German Diabetes Center, it turned out to be an offer we couldn't refuse. With our history of intense collaboration, Richard and I are both confident that the most fun is still ahead of us. And, Richard has a standing invitation for a sabbatical in the Bavarian Alps!

JCI: How important have these collaborations been to shaping your science?

Tschöp: The most important lesson I have learned is that interdisciplinary and translational teamwork is - at least for us key to every single breakthrough. It was painful at times, since we all speak different languages (literally and figuratively!) but it always paid off.

\section{Kathryn Claiborn}

